\title{
3D Prototype of Limited Lean Suspension System
}

\author{
G. Shiva Guru Prakash, Srinivaas Ashok Kumar
}

\begin{abstract}
D$ printing is also known as Additive manufacturing $(A M)$ process, where $3 D$ objects are created by laying down into successive layer of material. In 1984, Charles Hull developed the technology of $3 D$ printing from digital data and named the technique as Stereo lithography (STL) in 1986. In this project, create the prototype of Limited Lean Suspension System by using Fused Deposition Modeling (FDM) process in which the CAD model is designed in Catia v5 software and converted to STL format. The STL format is uploaded to FDM printer and works according to the G-code generated. This prototype is to study the kinematic parameters, working principle and in future research. By using Leaning application it will improves the vehicle stability, smooth cornering and fuel efficiency.
\end{abstract}

Keywords: CAD model, AM, STL, 3D Printing, Catia, Suspension.

\section{INTRODUCTION}

India is one of the developing countries which created more impact by using advanced technology called as 3D printing which converts virtual data into physical object. 3D printing is also known as Additive manufacturing (AM) process, where $3 \mathrm{D}$ objects are created by laying down into successive layer of material. In 1984, Charles Hull developed the technology of 3D printing from digital data and named the technique as Stereo lithography (STL) in 1986. There are lot of applications in 3D printing which was widely used by Industrial designers and engineers like Medicines, Household products, Automobile, Aerospace and Aircrafts. There are 3 fundamental manufacturing processes for 3D structure like Additive, Subtractive and Formative. The categories of 3D process are shown in Figure 1.

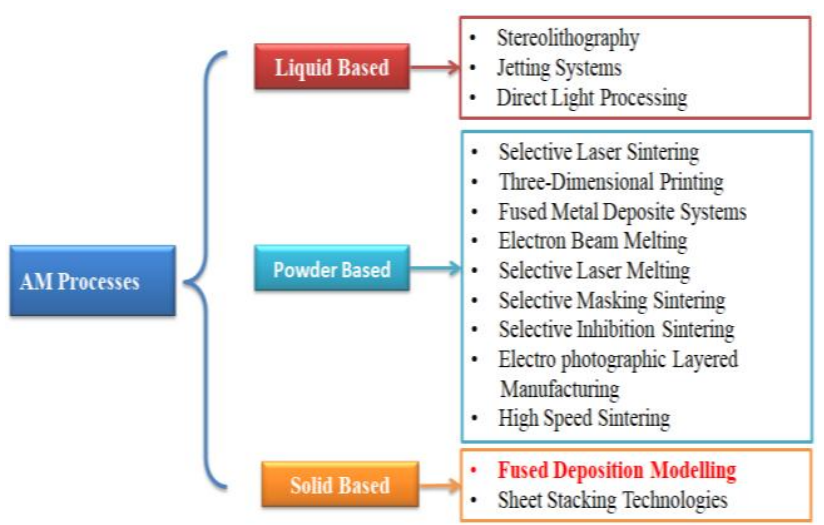

Fig1: Category of AM process

Revised Manuscript Received on July 10, 2019

G. Shiva Guru Prakash, Student, Dept. of Mechanical Engineering, Amrita School of Engineering, Amrita Vishwa Vidyapeetham, Amrita University, Coimbatore, T.N, India. (Email: shivaguru2k11@gmail.com)

Srinivaas Ashok Kumar, Assistant Professor, Dept. of Mechanica Engineering, Amrita School of Engineering, Amrita Vishwa Vidyapeetham, Amrita University, Coimbatore, T.N, India. (Email: a_srinivaas@cb.amrita.edu)
In this project, Fused Deposition Modeling (FDM) process is used which are widely used by most of the industries in order to eliminate the wastage of material, cost effective, ease of processing, good layer adhesion and high flexibility. In 1989, S. Scott and Lisa Crump patented the FDM technology. In this technology different kinds of filaments are used to create 3D objects like,

- Acrylonitrile Butadiene Styrene (ABS);

- Polylactic Acid (PLA);

- $\quad$ Polyethylene Terephthalate (PET);

- Nylon PA12;

- Polycarbonate (PC)

But here Polylactic Acid (PLA) filament is used for this process where this filament forms good layer adhesion, surface quality, strength \& stiffness and it is less cost. In this process, the plastic filament feeds into a heated extruder block, where it is heated and pushed through a nozzle into printing area and forms successive layer to obtain the final shape. The FDM printer and filament set up as shown in Figure 2.
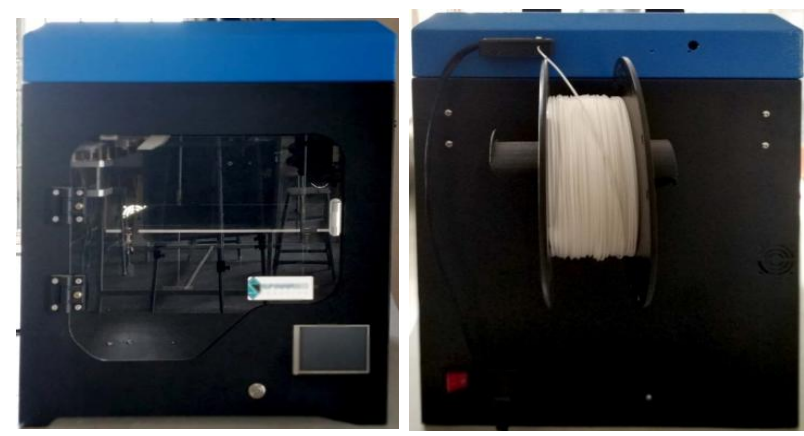

Fig2: FDM Printer

Ismayuzri B. Ishak, Mark B. Moffett \& Pierre Larochelle, published a paper algorithm for generating $3 \mathrm{~d}$ lattice structures suitable for printing on a multi-plane FDM printing platform. In this paper, describes the fabrication of multi-plane geometry (complex) by using FDM technology which shows the result comparing with traditional material removal process with 3D printing process gives better geometry and low material wastage in FDM process [4].

Pok Yin Victor Leung published a paper on Sugar 3D Printing:

Additive

Manufacturing with Molten Sugar for 
3D PROTOTYPE OF LIMITED LEAN SUSPENSION SYSTEM

Table1: Comparison of properties of filament material

\begin{tabular}{lccccc}
\hline Material & PLA & ABS & PET & Nylon PA12 & PC \\
\hline Density [g/cm3] & 1.24 & 1.05 & 1.27 & 1.25 & 1.2 \\
Water absorption [\%] & --- & 0.85 & 0.13 & 1.1 & 0.35 \\
Elastic modulus [Mpa] & 3310 & 2500 & 2100 & 1900 & 2300 \\
Tensile strength [Mpa] & 110 & --- & 50 & 42 & 60 \\
Print Temperature [ $\left.{ }^{\circ} \mathrm{C}\right]$ & 190 & 245 & 225 & 255 & 285 \\
\hline
\end{tabular}

Investigating Molten Material Fed Printing. In this paper, proposed that manufacturing of molten glass in 3D printing by using mixture of sucrose sugar and corn syrup which maintained at temperature range of $\left(100{ }^{\circ} \mathrm{C}-150{ }^{\circ} \mathrm{C}\right)$. This shows that by using machines with new techniques which require more temperature around $1000{ }^{\circ} \mathrm{C}$ when compare to 3D printing, it needs less temperature and also less cost [5]. Thipachart Punyaratabandhu, Peter C. Liacouras, and Sutipat Pairojboriboon, published a paper on using 3D models in orthopedic oncology: presenting personalized advantages in surgical planning and intra operative outcomes. The scope of this paper is to defining the geometry for medical application like tumor mass, bone loss and nearby vessels to determine most accurate osteotomy site and the most appropriate prosthesis which reduces the operative time and blood loss for the patient [7]. Igor Skawinski and Tomasz G. Grabowski published a paper on FDM 3D printing method utility assessment in small RC aircraft design. This paper investigated the possibility of manufacturing the wings for aircraft by using FDM process which shows that high reliability, low wastage of material and more flexibility. This kind of technique can be useful for quick and cost effective prototypes [9].

In this project, developing the prototype of Limited Lean Suspension System in order to improve the Kinematic parameters of the Vehicle and also improves the stability of the vehicle. The prototype was manufactured by using Fused Deposition Modeling (FDM) method. This method is commonly used for ease of manufacturing, low cost, and also more flexibility. In this technology, thermoplastic polymer is used as Polylactic acid (PLA) which provides good mechanical properties like thickness, surface quality and also provides good layer adhesion. PLA filament is fed to extruder block, where it is heated and pushed through a nozzle into the printing area. This steps repeats until the final shape is obtained. The nozzle diameter is $0.4 \mathrm{~mm}$ which provided good surface quality and flexibility.

\section{GENERIC AM PROCESS}

Here, Fused Deposition Modeling (FDM) process is used to build the prototype and PLA filament is used as a thermoplastic polymer based on the requirement. To carryon the process, there are certain steps need to follow:

- Pre-processing - preparing the 3D model

- Processing - build the 3D part in 3D printer

- Post-processing - removing / cleaning the part and Assembling

\section{PRE-PROCESSING}

Step1: preparing the CAD model

To build a prototype, the CAD model is the first and foremost step for developing the prototype. This is visualization phase. There are certain software's to create 3D model like Solid works, Parts inventory, Creo, Catia and so on. Here, Catia v5 software is used for CAD model and it takes nearly 25 days to complete the model by considering arbitrary values for some parts and assembled according to the requirement and also depends on the printing technology. According to the printing technology, the angle of over hangs should be minimized due to support generation.

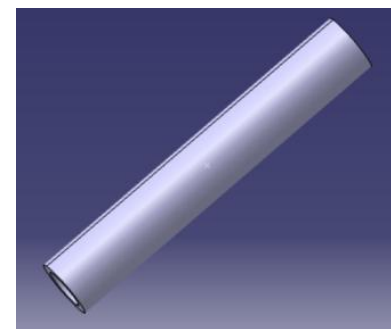

Fig3: CAD Part

Step2: STL conversion

After the CAD part is modeled the very next step is to convert the part file to STL file format. The 3D printer supports two types of file format like OBJ \& STL file format. Most of the user commonly used as STL format where every printer supports and also it's a standard format which describes the surface of the object by using polygons. STL has several acronyms such as Standard Triangle Language and Stereolithography Tessellation Language. OBJ file format is rarely used by user who needs texture maps, color and other details of CAD model which is stored in it. Even though STL file format is preferred for the entire $3 \mathrm{D}$ printer for ease of use.

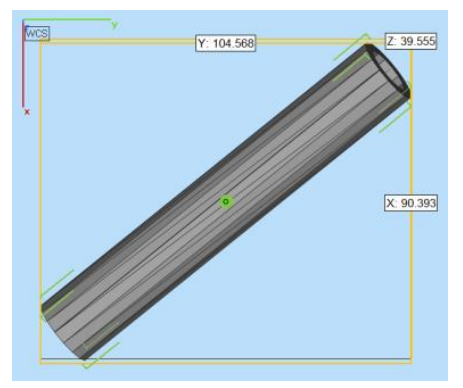

Fig4: STL formatted part

Step3: Uploading the part to the printer

Once a STL file format is generated, the file is imported to slicer program (Simplify 3D). The slicer program slices the part into horizontal layer according to the support 
generation, calculates the time needed to print the part and amount of material used. It converts the instruction into Gcode; this code is read by the printer and works accordingly in all 3 axes $(\mathrm{X}, \mathrm{Y} \& \mathrm{Z})$. The surface quality and Layer adhesion mainly depends on the slicer settings and printer set-up.

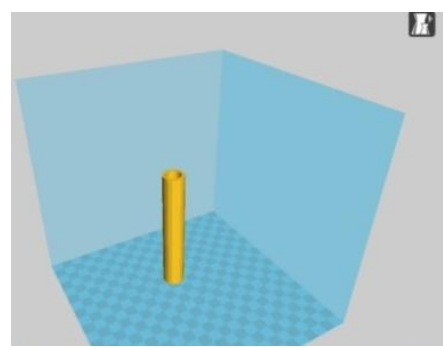

Fig5: Slicer Set-up

\section{PROCESSING}

Step4: Printer set-up

After all the slicer settings and support generation, the printer as to setup according to the requirement by maintaining the bed temperature $\left(60^{\circ} \mathrm{C}\right)$, cleaning the bed surface, nozzle tip and filament requirement depend upon the part. The filament used as PLA thermoplastic polymer based on the requirement.

Table2: Printer set-up

\begin{tabular}{|l|l|l|}
\hline \multirow{2}{*}{ Quality } & Layer thickness $(\mathrm{mm})$ & 0.2 \\
\cline { 2 - 3 } & Shell thickness $(\mathrm{mm})$ & 0.8 \\
\hline \multirow{3}{*}{ Fill } & Bottom / Top thickness $(\mathrm{mm})$ & 0.6 \\
\cline { 2 - 3 } Speed \& & Fill Density $(\%)$ & 20 \\
\hline \multirow{3}{*}{ Semperature } & Print speed $(\mathrm{mm} / \mathrm{s})$ & 50 \\
\cline { 2 - 3 } & Printing temperature $\left({ }^{\circ} \mathrm{C}\right)$ & 210 \\
\cline { 2 - 3 } & Bed temperature $\left({ }^{\circ} \mathrm{C}\right)$ & 60 \\
\hline \multirow{3}{*}{ Filament } & Support type & Everywhere \\
\cline { 2 - 3 } & Platform adhesion type & Raft \\
\cline { 2 - 3 } Machine & Diameter $(\mathrm{mm})$ & 1.75 \\
\cline { 2 - 3 } & Flow $(\%)$ & 100 \\
\hline
\end{tabular}

Step5: Part manipulation and build

After the printer setup the STL format is uploaded to the printer which reads the G-code instruction and works accordingly. It creates layer by layer formation horizontally on the bed surface which is maintained at $60{ }^{\circ} \mathrm{C}$ until the final part is obtained.

\section{POST PROCESSING}

Step6: Remove and cleaning the part

After the part is built by FDM printer according to the setup, the nozzle tip will automatically back to the original position and kept the part for cooling to room temperature for few minutes. After cooled down, the part was removed from the printer and remove the support generated and unwanted material from the part. To improve the surface finish, emery sheet of various grid sizes is used to remove the outer layer of the part which improves the surface finish and clean the rubbed material from the part and surface.

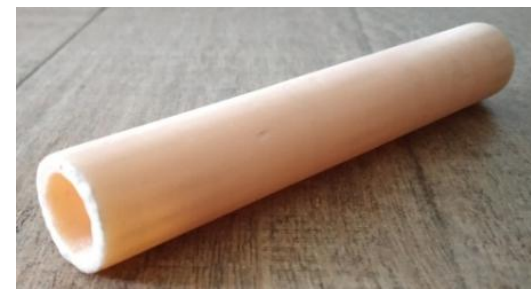

Fig6: Post processing part

\section{3D PRINTED PARTS}

The CAD model designed from the CATIA v5 software which includes 13 different parts. All the parts are converted to STL format and setup the printer and slicer. The STL file is uploaded to the printer which reads the G-code and works accordingly. To print all the parts in FDM technology it takes 20-25 days to complete.

1. Base

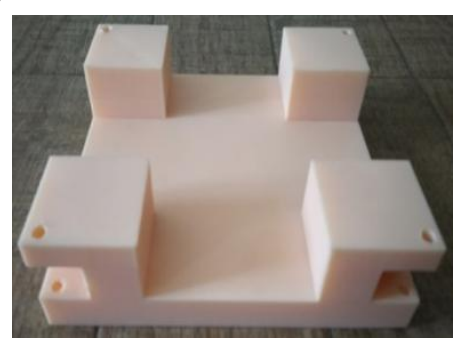

2. Main Frame

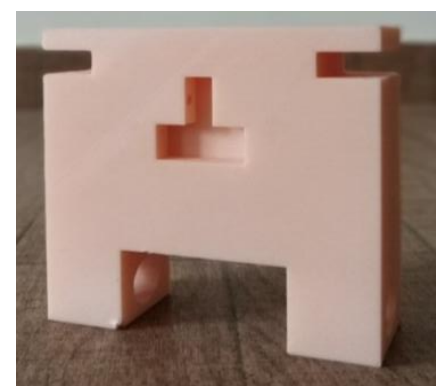

3. Hooks

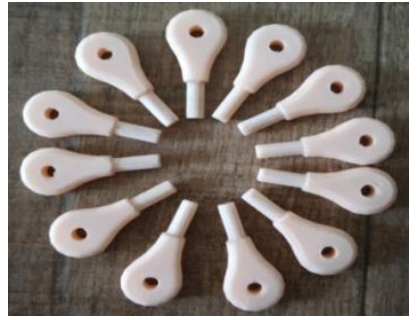

4. Bush

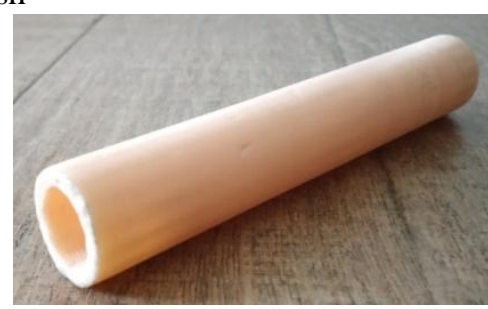

5. Pins 


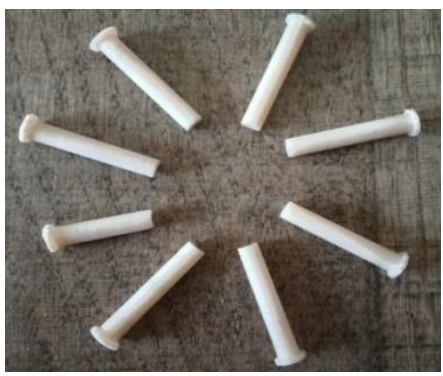

6. Side support

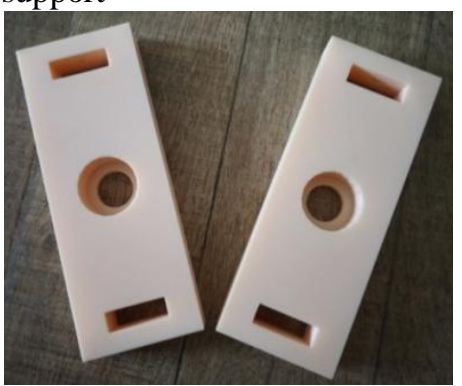

7. Suspensions

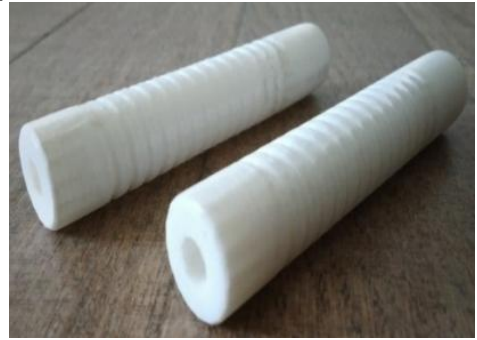

8. Gas cylinder

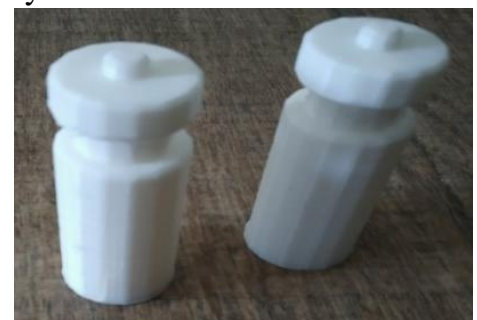

9. Rod \& Clip

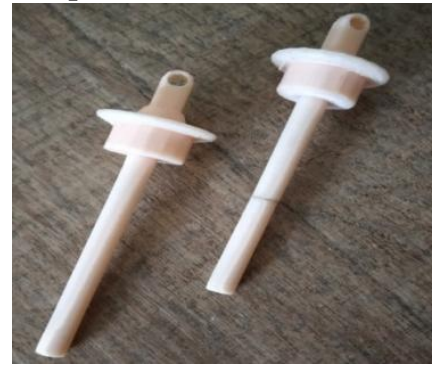

10. Bearings

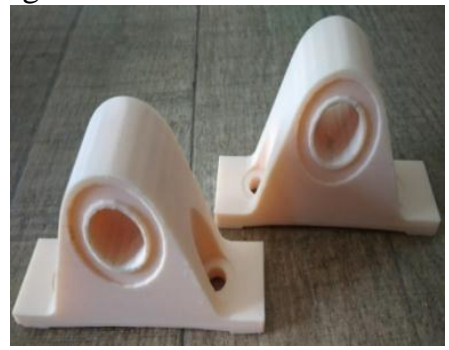

11. Lower Control Arm (LCA)

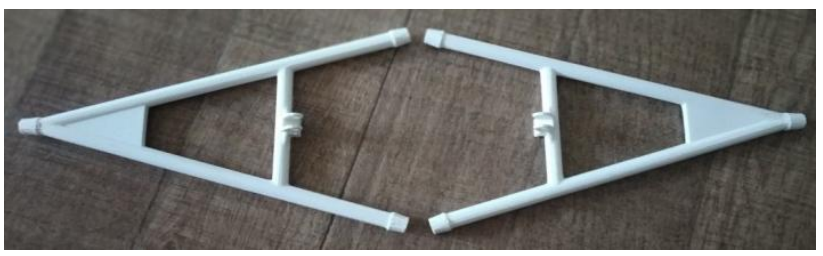

12. Upper Control Arm (UCA)

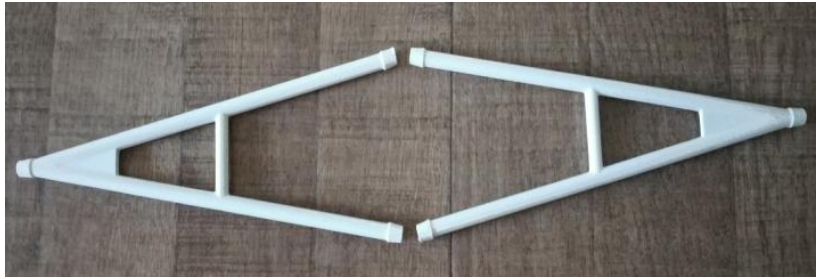

Fig7: All printed parts by FDM process

\section{LEANINIG SUSPENSION}

Basically, the suspension includes linkages, springs and shock absorber which helps to reduce the vibration of the vehicle and improves the passenger's comfort and ride ability.

In normal suspension, the vehicle front suspension parameters are affected due to cornering, improper wheel alignment, and tire radius, which results in passengers will get uncomforted, loss steering control, braking effect, rollover of vehicle and loss of traction due to the wheel is not perpendicular to the road in cornering. In most of the manufacturer will not consider the kinematic parameters as important.

In leaning Suspension, the vehicle front suspension parameters could improve by certain lean angle of the wheels, which the tire will remain perpendicular to the road while cornering. It will also increase the performance, safety

and economy of vehicles. To design the leaning suspension, a particular suspension system should be considered to meet the requirements and should also effective in use.

\section{OBJECTIVES}

1. Study the static and dynamic parameters of the front vehicle suspension.

2. Study the existing suspension system and parameters affecting its performance.

3. Improve the passenger's safety and comfort.

4. Improve the stability and cornering of the vehicle.

5. Study the wheel alignment and tire contact patch of the vehicle.

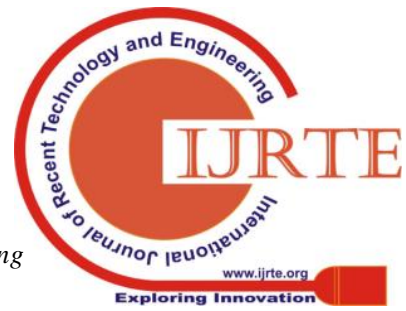




\section{DESIGN OF LEANING SUSPENSION}

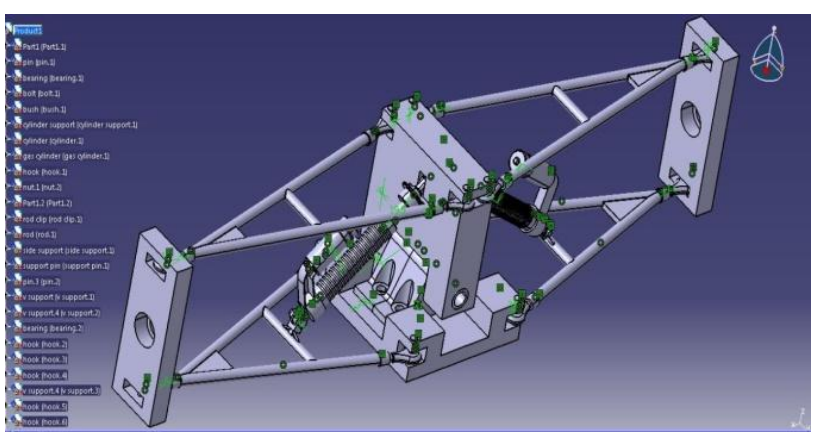

Fig8: 3D view of Leaning suspension

\section{RESULTS \& DISCUSSION}

After all the parts are printed and removed the unwanted material, the very next step is to assemble all the parts and define the parameters and working principle of the system. The parameters like surface finish, flexibility and strength of the parts to be defined and also the working procedure of the system as shown. The obtained 3D part from Printer is assembled and compared with CAD model designed from Catia v5. Both the assembled model is exactly similar to the designed one and operated the $3 \mathrm{D}$ prototype, which is flexible and high strength. The CAD model design and 3D printed model are shown below.

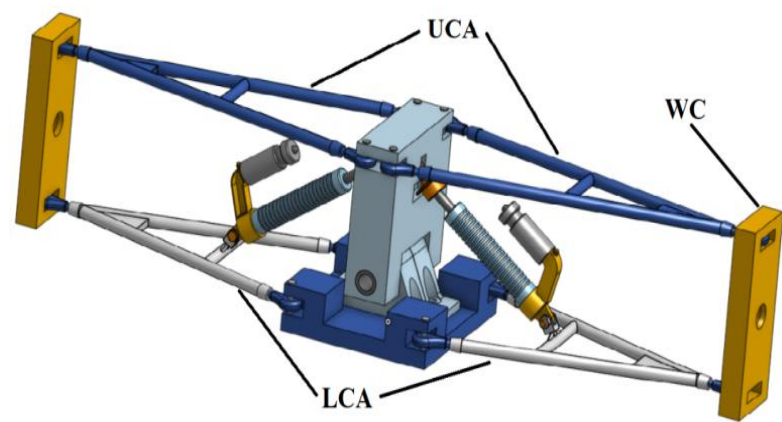

Fig9: CAD model design from Catia

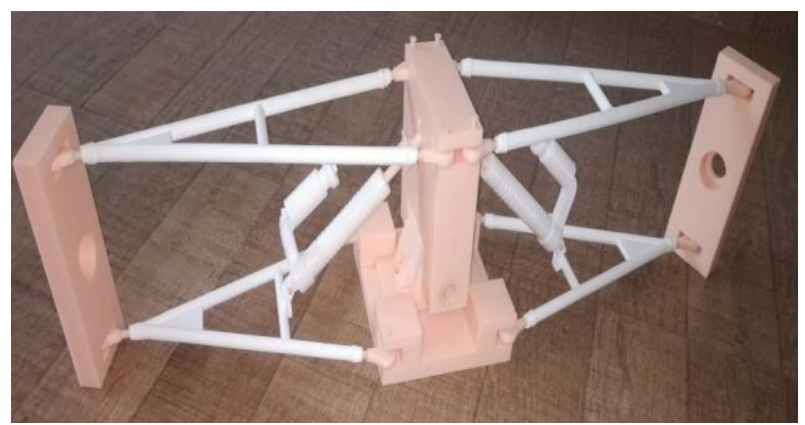

Fig10: 3D prototype from FDM Printer

\section{CONCLUSION}

In this project we studied the kinematic parameters of the Suspension system inorder to improve the efficiency and stability of the vehicle. The CAD model is designed in Catia v5 and converted the file into STL format. The converted file is uploaded to the printer and assembled after the parts printed. The assembled 3D model is shown below and operated in according to the principle. The model is flexible to operate and also cost effective, which reduces the wastage of material and also it can used for research.

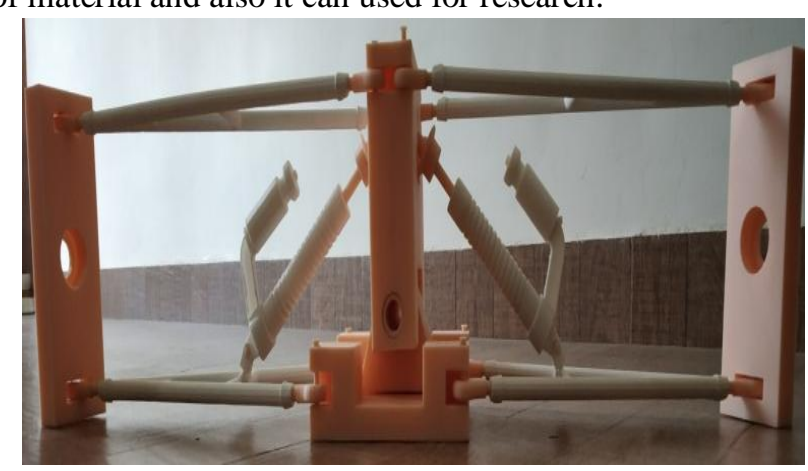

Fig11: Assembled 3D prototype

\section{REFERENCES}

1. Martin Schaich et al., "3D Documentation of the Igel Column Original and Copy". 978-1-4799-3170-5/13/\$31.00 (C2013 IEEE.

2. Li Tang et al., "Algorithm for 3D reconstruction with both visible and missing data". ELECTRONICS LETTERS, 13th November 2003 Vol. 39 No. 23.

3. Meng Yao-wei \& Wang Shan-dong, "A New Interactive Approach of 3D Modeling Reconstruction from Contours". 20 IO International Conference on Computer Application and System Modeling (ICCASM 2010).

4. Ismayuzri B. Ishak, Mark B. Moffett \& Pierre Larochelle, "An Algorithm for generating 3d lattice structures suitable for printing on a multi-plane FDM printing platform". Proceedings of the ASME 2018, IDETC/CIE 2018, August 26-29, 2018, Canada.

5. Pok Yin Victor Leung, "Sugar 3D Printing: Additive Manufacturing with Molten Sugar for Investigating Molten Material Fed Printing". 3D PRINTING AND ADDITIVE MANUFACTURING, Volume 4, Number 1, 2017.

6. F.F. Pieterse and A.L. Nel, "The advantages of 3D printing in undergraduate Mechanical Engineering research". 2016 IEEE Global Engineering Education Conference (EDUCON), 10-13 April 2016, Abu Dhabi, UAE.

7. Punyaratabandhu et al., "3D Printing in Medicine", (2018) 4:12, [doi.org/10.1186/s41205-018-0035-6].

8. Chee Kai Chua et al., "Lasers in 3D printing and Manufacturing". World scientific, [doi/10.1142/9789814656436_0005].

9. Igor Skawinski and Tomasz G. Grabowski, "FDM 3D printing method utility assessment in small RC aircraft design". Emerald Publishing Limited [ISSN 1748-8842] [DOI 10.1108/AEAT-07-2018-0189].

10. B. Coppola et al., "Investigation on the use of PLA/hemp composites for the fused deposition modelling (FDM) 3D printing". 9th International Conference on "Times of Polymers and Composites", [doi.org/10.1063/1.5045948].

11. Yah Yun Aw et al., "Effect of Printing Parameters on CABS/ZnO Composites". Materials 2018, 11, 466; [doi:10.3390/ma11040466].

12. AHM Haidiezul, AF Aiman and B Bakar, "Surface Finish Effects Using Coating Method on 3D Printing (FDM) Parts". IOP Conf. Series: Materials Science and Engineering, 318 (2018) 012065; [doi:10.1088/1757-899X/318/1/012065]. 